\title{
Sparse Representation for Target Detection in Hyperspectral Imagery
}

\author{
Yi Chen, Nasser M. Nasrabadi, Fellow, IEEE, and Trac D. Tran, Senior Member, IEEE
}

\begin{abstract}
In this paper, we propose a new sparsity-based algorithm for automatic target detection in hyperspectral imagery (HSI). This algorithm is based on the concept that a pixel in HSI lies in a low-dimensional subspace and thus can be represented as a sparse linear combination of the training samples. The sparse representation (a sparse vector corresponding to the linear combination of a few selected training samples) of a test sample can be recovered by solving an $\ell_{0}$-norm minimization problem. With the recent development of the compressed sensing theory, such minimization problem can be recast as a standard linear programming problem or efficiently approximated by greedy pursuit algorithms. Once the sparse vector is obtained, the class of the test sample can be determined by the characteristics of the sparse vector on reconstruction. In addition to the constraints on sparsity and reconstruction accuracy, we also exploit the fact that in HSI the neighboring pixels have a similar spectral characteristic (smoothness). In our proposed algorithm, a smoothness constraint is also imposed by forcing the vector Laplacian at each reconstructed pixel to be minimum all the time within the minimization process. The proposed sparsity-based algorithm is applied to several hyperspectral imagery to detect targets of interest. Simulation results show that our algorithm outperforms the classical hyperspectral target detection algorithms, such as the popular spectral matched filters, matched subspace detectors, adaptive subspace detectors, as well as binary classifiers such as support vector machines.
\end{abstract}

Index Terms-Hyperspectral imagery, sparse recovery, sparse representation, spatial correlation, target detection.

\section{INTRODUCTION}

$\mathbf{H}$ YPERSPECTRAL remote sensors capture digital images in hundreds of narrow spectral bands (about $10 \mathrm{~nm}$ wide), which span the visible to infrared spectrum [1]. Pixels in HSI are represented by $B$-dimensional vectors where $B$ is the number of spectral bands. Different materials are usually assumed to be spectrally separable as they reflect electromagnetic energy differently at specific wavelengths. This property enables discrimination of materials based on the radiance spectrum obtained

Manuscript received April 20, 2010; revised September 24, 2010 and December 20, 2010; accepted January 31, 2011. Date of publication February 10, 2011; date of current version May 18, 2011. This paper was supported in part by the Army Research Office (ARO) under Grant 58110-MA-II and in part by the National Science Foundation (NSF) under Grant CCF-0728893. The associate editor coordinating the review of this manuscript and approving it for publication was Dr. Gustavo Camps-Valls

Y. Chen and T. D. Tran are with the Department of Electrical and Computer Engineering, The Johns Hopkins University, Baltimore, MD 21218 USA (e-mail: ychen98@jhu.edu; trac@jhu.edu).

N. M. Nasrabadi is with the U.S. Army Research Laboratory, Adelphi, MD 20783 USA (e-mail: nnasraba@arl.army.mil).

Color versions of one or more of the figures in this paper are available online at http://ieeexplore.ieee.org.

Digital Object Identifier 10.1109/JSTSP.2011.2113170 by hyperspectral imagery. HSI has found many applications in various fields such as military [2]-[4], agriculture [5], [6], and mineralogy [7]. One of the important applications of HSI is target detection, which can be viewed as a two-class classification problem where pixels are labeled as target (target present) or background (target absent) based on their spectral characteristics. Support vector machines [8], [9] have been a powerful tool to solve supervised classification problems and have shown a good classification performance for hyperspectral classification [10], [11]. A number of algorithms also have been proposed for target detection in HSI based on statistical hypothesis testing techniques [2]. Among these approaches, spectral matched filters [12], [13], matched subspace detectors [14], and adaptive subspace detectors [15] have been widely used to detect targets of interests. The details of these classical algorithms will be described in the next section.

Recently, a novel signal classification technique via sparse representation have been proposed for face recognition [16]. It is observed that aligned faces of the same object with varying lighting conditions approximately lie in a low-dimensional subspace [17]. Thus, a test face image can be sparsely represented by training samples from all classes. The most compact representation can be obtained by solving a sparsity-constrained optimization problem. This algorithm exploits the discriminative nature of sparse representation and the reconstruction of the test sample provides directly its classification label. This idea naturally extends to other signal classification problems such as iris recognition [18], tumor classification [19], and HSI unmixing [20].

In this paper, we propose a target detection algorithm based on sparse representation for HSI data. We use the same sparsity model in [16] where a test sample is approximately represented by very few training samples from both target and background dictionaries, and the recovered sparse representation is used directly for detection. In addition to the constraints on sparsity and reconstruction accuracy, we show that it is necessary to exploit the fact that neighboring HSI pixels usually have a similar spectral characteristics as well. To achieve this, we impose a smoothing constraint on the reconstructed image by forcing the vector Laplacian, as defined in Section III-D, of the reconstructed pixels to be zero. By incorporating this spatial correlation, the detection performance is significantly improved for images in which targets consist of multiple pixels.

One of the advantages of our proposed approach is that there is no explicit assumption on the statistical distribution characteristics of the observed data as in the previous target detection algorithms [12]-[15]. Furthermore, in the spectral matched filter, the target spectral signature is a single vector, usually obtained 
by averaging the training target samples or from a spectral library. However, using a single target spectrum is usually insufficient to represent the target spectral characteristics since the target spectrum changes with the environmental situation. This problem can be avoided by using a target subspace model represented by training samples that account for the target spectrum under various conditions of illumination and atmospheric conditions, making the dictionary invariant to the environmental variations [21], [22]. This environmental invariant approach can easily be incorporated into our algorithm by augmenting the target and background dictionaries with synthetically generated spectral signatures in order to construct better target and background subspaces. Moreover, unlike the other detectors based on statistical hypothesis testing, the sparsity model in our approach has the flexibility of imposing additional restrictions corresponding to the characteristics of HSI such as smoothness across neighboring hyperspectral pixels.

The paper is structured as follows. Section II briefly describes several previously proposed approaches commonly used in automatic target detection in HSI. Our sparsity-driven target detection algorithm is presented in Section III. The effectiveness of the proposed method is demonstrated by simulation results presented in Section IV. Conclusions are drawn in Section V. Throughout this paper, matrices and vectors are denoted by upper and lower case boldface letters, respectively.

\section{PREVIOUS APPROACHES}

In this section, we briefly introduce previously developed approaches for target detection in HSI. Specifically, we describe problem formulation of support vector machines (SVMs), followed by the signal models and detector expressions of the classical detectors including spectral matched filter (SMF), matched subspace detectors (MSDs), and adaptive subspace detectors (ASDs). Implementation details of the three statistical detectors and their nonlinear (kernel) versions can be found in [23], whereas details of SVM can be found in [24].

\section{A. Support Vector Machines}

The SVM approach [8] solves the supervised binary classification problem by seeking the optimal hyperplane that separates two classes with the largest margin. A nonlinear SVM (called kernel SVM) is often implemented to further improve the separation between classes by projecting the samples onto a higher dimensional feature space. In kernel SVM, the dot products in the original SVM formulation are replaced by a nonlinear kernel function using the kernel trick [8].

It has also been shown that the integration of the contextual information via composite kernels in SVM (i.e., contextual SVM) leads to an improvement in HSI classification over the traditional spectral-only SVM [24], [25]. In contextual SVM, a pixel $\boldsymbol{x}_{i}$ is redefined as a combination of the spectral pixel $\boldsymbol{x}_{i}^{w}$ and its spatial feature $\boldsymbol{x}_{i}^{s}$ (e.g., the mean and standard deviation per spectral band) extracted in a small neighborhood. In this paper, we implemented contextual SVM with a composite kernel that fuses the spectral and spatial information via a weighted summation

$$
K\left(\boldsymbol{x}_{i}, \boldsymbol{x}_{j}\right)=\mu K_{s}\left(\boldsymbol{x}_{i}^{s}, \boldsymbol{x}_{j}^{s}\right)+(1-\mu) K_{w}\left(\boldsymbol{x}_{i}^{w}, \boldsymbol{x}_{j}^{w}\right)
$$

where $\mu \in[0,1]$ is the tradeoff between spatial kernel $K_{s}$ and spectral kernel $K_{w}$. Examples of possible kernels can be found in [26].

\section{B. Spectral Matched Filter}

Let $\boldsymbol{x}=\left[\begin{array}{llll}x_{1} & x_{2} & \cdots & x_{B}\end{array}\right]^{T}$ be a spectral observation consisting of $B$ spectral bands. The model for SMF can be expressed by

$$
\begin{aligned}
& H_{0}: \boldsymbol{x}=\boldsymbol{n}, \quad \text { target absent } \\
& H_{1}: \boldsymbol{x}=a \boldsymbol{s}+\boldsymbol{n}, \quad \text { target present }
\end{aligned}
$$

where $a$ is the target abundance measure ( $a=0$ when no target is present and $a>0$ when a target is present), $\boldsymbol{s}=$ $\left[\begin{array}{llll}s_{1} & s_{2} & \cdots & s_{B}\end{array}\right]^{T}$ is the spectral signature of the target, and $\boldsymbol{n}$ is the additive background noise.

Assume $\boldsymbol{n}$ is zero-mean Gaussian random noise. Using the generalized likelihood ratio test (GLRT), the output of SMF for a test input $\boldsymbol{x}$ is given by [12]

$$
D_{\mathrm{SMF}}(\boldsymbol{x})=\frac{\boldsymbol{s}^{T} \hat{\boldsymbol{C}}^{-1} \boldsymbol{x}}{\boldsymbol{s}^{T} \hat{\boldsymbol{C}}^{-1} \boldsymbol{s}}
$$

where $\hat{\boldsymbol{C}}$ represents the estimated covariance matrix for the centered observation data. If the output $D_{\mathrm{SMF}}(\boldsymbol{x})$ is greater than a prescribed threshold $\delta$, then the test sample will be determined as a target; otherwise, it will be labeled as background.

Variations of SMF include the adaptive SMF (ASMF) where the background clutter covariance matrix is estimated from a small number of samples in the neighborhood of the test sample and the regularized SMF [27] where a regularization term is added to force the filter coefficients to shrink and become smooth. The regularized SMF is implemented in Section IV for detector performance comparison.

\section{Matched Subspace Detectors}

In the previous SMF approach, only a single target spectral signature is used. However, in MSD, a pixel is modeled in terms of target subspace and background subspace which are obtained using target and background training data, respectively. The target detection set-up for MSD is

$$
\begin{aligned}
& H_{0}: \boldsymbol{x}=\boldsymbol{B} \boldsymbol{\zeta}+\boldsymbol{n}, \quad \text { target absent } \\
& H_{1}: \boldsymbol{x}=\boldsymbol{T} \boldsymbol{\theta}+\boldsymbol{B} \boldsymbol{\zeta}+\boldsymbol{n}, \quad \text { target present }
\end{aligned}
$$

where $B$ and $\boldsymbol{T}$ represent matrices whose columns are linearly independent and span the background and target subspaces, respectively; $\boldsymbol{\zeta}$ and $\boldsymbol{\theta}$ are unknown vectors whose entries are coefficients accounting for the abundances of the corresponding column vectors of $\boldsymbol{B}$ and $\boldsymbol{T}$, respectively; and $\boldsymbol{n}$ is additive Gaussian noise.

The GLRT for the above model is [14]

$$
D_{\mathrm{MSD}}(\boldsymbol{x})=\frac{\boldsymbol{x}^{T}\left(\boldsymbol{I}-\boldsymbol{P}_{\boldsymbol{B}}\right) \boldsymbol{x}}{\boldsymbol{x}^{T}\left(\boldsymbol{I}-\boldsymbol{P}_{\boldsymbol{T} B}\right) \boldsymbol{x}}
$$

where $\boldsymbol{P}_{\boldsymbol{B}}$ is the projection matrix associated with the background subspace $\langle\boldsymbol{B}\rangle$, and $\boldsymbol{P}_{\boldsymbol{T} B}$ is the projection matrix associated with the target-and-background subspace $\langle\mathbf{T B}\rangle$. Usually, the eigenvectors corresponding to the significant eigenvalues of 
the target and background covariance matrices are used to generate the columns of $T$ and $B$, respectively. For a prescribed threshold $\delta$, if the output $D_{\mathrm{MSD}}(\boldsymbol{x})>\delta$, then $\boldsymbol{x}$ will be labeled as target; otherwise, it will be labeled as background.

\section{Adaptive Subspace Detectors}

A scaled background noise under $H_{1}$ is used in ASD because in the case of subpixel targets, the amount of background covered area may be different from that of a pure background pixel. For ASD, the detection model for a measurement $\boldsymbol{x}$ is

$$
\begin{aligned}
& H_{0}: \boldsymbol{x}=\boldsymbol{n}, \quad \text { target absent } \\
& H_{1}: \boldsymbol{x}=\boldsymbol{U} \boldsymbol{\theta}+\sigma \boldsymbol{n}, \quad \text { target present }
\end{aligned}
$$

where $\boldsymbol{U}$ is a matrix whose columns are linearly independent vectors that span the target subspace $\langle\boldsymbol{U}\rangle, \boldsymbol{\theta}$ is an unknown vector of the abundances of the corresponding columns of $\boldsymbol{U}$, $\boldsymbol{n}$ is Gaussian random noise, and $\sigma$ is a scalar. The measurement $\boldsymbol{x}$ is assumed to be background noise under hypothesis $H_{0}$ and a linear combination of a target subspace signal and scaled background noise under hypothesis $H_{1}$.

The GLRT for the above problem is given by [15]

$$
D_{\mathrm{ASD}}(\boldsymbol{x})=\frac{\boldsymbol{x}^{T} \hat{\boldsymbol{C}}^{-1} \boldsymbol{U}\left(\boldsymbol{U}^{T} \hat{\boldsymbol{C}}^{-1} \boldsymbol{U}\right)^{-1} \boldsymbol{U}^{T} \hat{\boldsymbol{C}}^{-1} \boldsymbol{x}}{\boldsymbol{x}^{T} \hat{\boldsymbol{C}}^{-1} \boldsymbol{x}}
$$

where $\hat{\boldsymbol{C}}$ is the estimated background covariance. Similar to the cases of SMF and MSD, if $D_{\mathrm{ASD}}(\boldsymbol{x})>\delta$, then $\boldsymbol{x}$ will be declared as target; otherwise, it will be labeled as background.

\section{SPARSITY-BASED TARGET DETECTION}

In this section, we introduce the first sparsity-based HSI target detection algorithm by sparsely representing the test sample using a structured dictionary consisting of target and background training samples. We first describe the details of the sparse subspace model employed in the proposed algorithm, and then demonstrate its ability as a classifier.

\section{A. Sparsity Model}

Let $\boldsymbol{x}$ be a hyperspectral pixel observation, which is a $B$-dimensional vector whose entries correspond to responses to various spectral bands. If $\boldsymbol{x}$ is a background pixel, its spectrum approximately lies in a low-dimensional subspace spanned by the background training samples $\left\{\boldsymbol{a}_{i}^{b}\right\}_{i=1,2, \ldots, N_{b}}$. The pixel $\boldsymbol{x}$ can then be approximately represented as a linear combination of the training samples as follows:

$$
\begin{aligned}
& \boldsymbol{x} \approx \alpha_{1} \boldsymbol{a}_{1}^{b}+\alpha_{2} \boldsymbol{a}_{2}^{b}+\cdots+\alpha_{N_{b}} \boldsymbol{a}_{N_{b}}^{b} \\
& =\underbrace{\left[\begin{array}{llll}
\boldsymbol{a}_{1}^{b} & \boldsymbol{a}_{2}^{b} & \cdots & \boldsymbol{a}_{N_{b}}^{b}
\end{array}\right]}_{\boldsymbol{A}_{b}} \underbrace{\left[\begin{array}{llll}
\alpha_{1} & \alpha_{2} & \cdots & \alpha_{N_{b}}
\end{array}\right]^{T}}_{\boldsymbol{\alpha}} \\
& =A_{b} \boldsymbol{\alpha}
\end{aligned}
$$

where $N_{b}$ is the number of background training samples, $\boldsymbol{A}_{b}$ is the $B \times N_{b}$ background dictionary whose columns are the background training samples (also called atoms), and $\boldsymbol{\alpha}$ is an unknown vector whose entries are the abundances of the corresponding atoms in $\boldsymbol{A}_{b}$. In our model, $\boldsymbol{\alpha}$ turns out to be a sparse vector (i.e., a vector with only few nonzero entries). To better

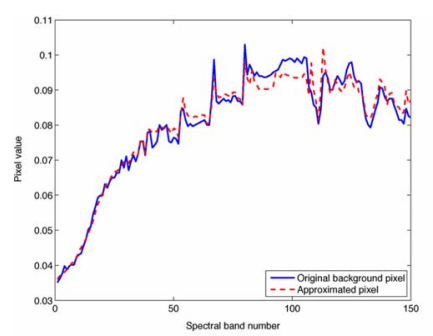

(a)

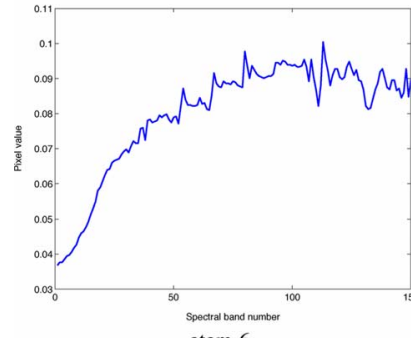

atom 6

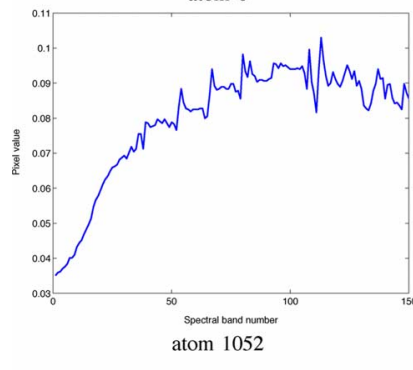

Fig. 1. Example of sparse representation of a background pixel. (a) The original pixel $\boldsymbol{x}$ (blue solid) and its approximation $\boldsymbol{A}_{b} \boldsymbol{\alpha}$ represented by four training samples in $\boldsymbol{A}_{b}$ (red dashed). The MSE between $\boldsymbol{x}$ and $\boldsymbol{A}_{b} \boldsymbol{\alpha}$ is $7.25 \times 10^{-6}$. (b) The sparse representation $\boldsymbol{\alpha}$ of $\boldsymbol{x}$. (c) The four background training spectral signatures corresponding to the non-zero entries of $\boldsymbol{\alpha}$.

illustrate this model, an example is shown in Fig. 1. A background sample $\boldsymbol{x}$ consisting of $B=150$ bands (blue solid) and its approximation $\boldsymbol{A}_{b} \boldsymbol{\alpha}$ (red dashed) are shown in Fig. 1(a). The background dictionary contains $N_{b}=1300$ training samples which are randomly picked from the entire image including spectral signature for multiple background materials (e.g., vegetation, dirt road, and soil). The sparse representation $\alpha$ is shown in Fig. 1(b). We see that only 4 out of the 1300 entries of $\boldsymbol{\alpha}$ are nonzero. The four atoms (background training samples) of $A_{b}$ corresponding to the nonzero entries are shown in Fig. 1(c). The test sample $\boldsymbol{x}$ is approximated by a linear combination of only four training atoms with a small reconstruction error of mean squared error $(\mathrm{MSE})=7.25 \times 10^{-6}$.

Similarly, a target pixel $\boldsymbol{x}$ approximately lies in the target subspace spanned by the target training samples $\left\{\boldsymbol{a}_{i}^{t}\right\}_{i=1,2, \ldots, N_{t}}$, which can also be sparsely represented by a linear combination of the training samples

$$
\begin{aligned}
& \boldsymbol{x}=\beta_{1} \boldsymbol{a}_{1}^{t}+\beta_{2} \boldsymbol{a}_{2}^{t}+\cdots+\beta_{N_{t}} \boldsymbol{a}_{N_{t}}^{t} \\
& =\underbrace{\left[\begin{array}{llll}
\boldsymbol{a}_{1}^{t} & \boldsymbol{a}_{2}^{t} & \cdots & \boldsymbol{a}_{N_{t}}^{t}
\end{array}\right]}_{\boldsymbol{A}_{t}} \underbrace{\left[\begin{array}{llll}
\beta_{1} & \beta_{2} & \cdots & \beta_{N_{t}}
\end{array}\right]^{T}}_{\boldsymbol{\beta}} \\
& =A_{t} \boldsymbol{\beta}
\end{aligned}
$$

where $N_{t}$ is the number of target training samples, $A_{t}$ is the $B \times N_{t}$ target dictionary consisting of the target training pixels, and $\boldsymbol{\beta}$ is a sparse vector whose entries contain the abundances 


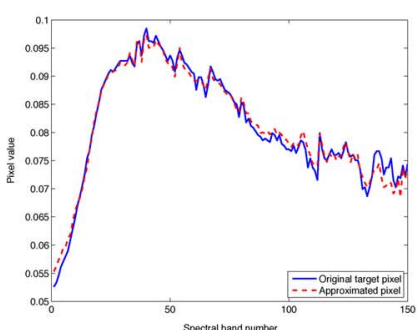

(a)
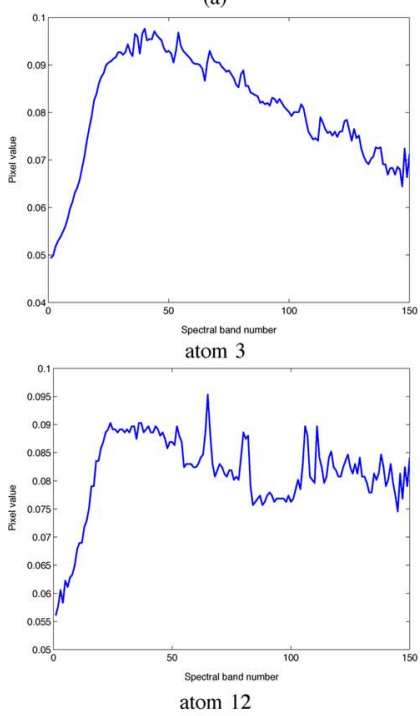

(c)

Fig. 2. Example of sparse representation of a target test sample. (a) The original pixel $\boldsymbol{x}$ (blue solid) and reconstructed pixel $\boldsymbol{A}_{t} \boldsymbol{\beta}$ represented by four training samples in $\boldsymbol{A}_{t}$ (red dashed). The MSE between $\boldsymbol{x}$ and $\boldsymbol{A}_{t} \boldsymbol{\beta}$ is $1.70 \times 10^{-6}$. (b) The sparse representation $\boldsymbol{\beta}$ of $\boldsymbol{x}$. (c) The four target training spectral signatures corresponding to the nonzero entries of $\beta$.

of the corresponding target atoms in $\boldsymbol{A}_{\boldsymbol{t}}$. An example demonstrating the effectiveness of this sparse-representation model is depicted in Fig. 2. The target dictionary has $N_{t}=18$ training samples. Note that because of the lack of availability of the target spectral signatures, the size of the training dictionary for targets is usually much smaller than that of the background dictionary. Fig. 2(a) shows the original target spectral (blue solid) and its approximation (red dashed) from four training atoms. The sparse vector $\boldsymbol{\beta}$ is shown in Fig. 2(b), and the atoms in $\boldsymbol{A}_{t}$ corresponding to the nonzero entries of $\beta$ are shown in Fig. 2(c).

In our proposed detection algorithm, an unknown test sample is modeled to lie in the union of the background and target subspaces. Therefore, by combining the two dictionaries $\boldsymbol{A}_{b}$ and $A_{t}$, a test sample $\boldsymbol{x}$ can be written as a sparse linear combination of all training pixels

$$
x=A_{b} \boldsymbol{\alpha}^{\prime}+A_{t} \beta^{\prime}=\underbrace{\left[\begin{array}{ll}
A_{b} & A_{t}
\end{array}\right]}_{A} \underbrace{\left[\begin{array}{c}
\alpha^{\prime} \\
\beta^{\prime}
\end{array}\right]}_{\gamma}=A \gamma
$$

where $\boldsymbol{A}=\left[\begin{array}{ll}\boldsymbol{A}_{b} & \boldsymbol{A}_{t}\end{array}\right]$ is a $B \times\left(N_{b}+N_{t}\right)$ matrix consisting of both background and target training samples, and $\gamma=\left[\begin{array}{ll}\boldsymbol{\alpha}^{\prime T} & \boldsymbol{\beta}^{\prime T}\end{array}\right]^{T}$ is a $\left(N_{b}+N_{t}\right)$-dimensional vector consisting of the two vectors $\boldsymbol{\alpha}^{\prime}$ and $\boldsymbol{\beta}^{\prime}$ associated with the two dictionaries. This model is similar to that of the MSD in (4) where the test sample is assumed to lie in a subspace spanned by training samples from both background and target classes. However, in the case of MSD, the target and background are assumed to have a Gaussian distribution and GLRT is used to develop the detector. In our sparsity-based model, no assumption about the target and background distributions is required. Also, in the MSD signal model, the columns of the background and target dictionaries have to be independent in order to generate the required projection operators. In our approach, the subspace model is more generalized since independence between the training samples is not necessary. The vector $\gamma$ is a concatenation of the two vectors associated with the background and target dictionaries and is also a sparse vector as follows. Since the background (e.g., trees, grass, road, soil) and target (e.g., metal, paint, glass) pixels usually consist of different materials, they have distinct spectral signatures and thus the spectrum of target and background pixels lie in different subspaces. For example, if $\boldsymbol{x}$ is a target pixel, then ideally it cannot be represented by the background training samples. In this case, $\boldsymbol{\alpha}^{\prime}$ is a zero vector and $\boldsymbol{\beta}^{\prime}$ is a sparse vector. On the other hand, if $\boldsymbol{x}$ belongs to the background class, then $\boldsymbol{\alpha}^{\prime}$ is sparse and $\boldsymbol{\beta}^{\prime}$ is a zero vector. Therefore, the test sample $\boldsymbol{x}$ can be sparsely represented by combined background and target dictionaries, and the locations of nonzero entries in the sparse vector $\gamma$ actually contains critical information about the class of the test sample $\boldsymbol{x}$. Next, we demonstrate how to obtain $\boldsymbol{\gamma}$ and how to label the class of a test sample from $\boldsymbol{\gamma}$.

\section{B. Reconstruction and Detection}

This section considers the reconstruction problem of finding the sparse vector $\boldsymbol{\gamma}$ for a test sample $\boldsymbol{x}$, given the dictionary $\boldsymbol{A}$. As discussed above, a test sample can be approximately represented by very few training samples. Given the dictionary of training samples $\boldsymbol{A}=\left[\begin{array}{ll}\boldsymbol{A}_{b} & \boldsymbol{A}_{t}\end{array}\right]$, the representation $\boldsymbol{\gamma}$ satisfying $\boldsymbol{A} \boldsymbol{\gamma}=\boldsymbol{x}$ can be obtained by solving the following optimization problem for the sparsest vector:

$$
\hat{\boldsymbol{\gamma}}=\arg \min \|\boldsymbol{\gamma}\|_{0} \quad \text { subject to } \quad \boldsymbol{A} \boldsymbol{\gamma}=\boldsymbol{x}
$$

where $\|\cdot\|_{0}$ denotes $\ell_{0}$-norm which is defined as the number of nonzero entries in the vector (also called the sparsity level of the vector). The above problem of minimizing the $\ell_{0}$-norm is a NP-hard problem. If the solution is sufficiently sparse, this NP-hard problem can be relaxed to a linear programming problem by replacing the $\ell_{0}$-norm by $\ell_{1}$-norm, which can then be solved efficiently by convex programming techniques [28], [29]. Alternatively, the problem in (11) can also be approximately solved by greedy pursuit algorithms such as orthogonal matching pursuit (OMP) [30] or subspace pursuit (SP) [31]. Due to the presence of approximation errors in empirical data, the equality constraint in (11) can be relaxed to an inequality one

$$
\hat{\boldsymbol{\gamma}}=\arg \min \|\boldsymbol{\gamma}\|_{0} \quad \text { subject to } \quad\|\boldsymbol{A} \boldsymbol{\gamma}-\boldsymbol{x}\|_{2} \leq \sigma
$$

where $\sigma$ is the error tolerance. The above problem can also be interpreted as minimizing the approximation error within a certain sparsity level

$$
\hat{\boldsymbol{\gamma}}=\arg \min \|\boldsymbol{A} \boldsymbol{\gamma}-\boldsymbol{x}\|_{2} \quad \text { subject to } \quad\|\boldsymbol{\gamma}\|_{0} \leq K_{0}
$$


where $K_{0}$ is a given upper bound on the sparsity level [32]. In [33], it has been shown that the solutions to the problems in (12) and (13) coincide for properly chosen parameters $\sigma$ and $K_{0}$, and therefore the two problems are in some sense equivalent. In this paper, the greedy SP algorithm [31] is used to approximately solve the sparse recovery problem (13) due to its computational efficiency.

The sparse vector $\hat{\gamma}$ is recovered by decomposing the pixel $\boldsymbol{x}$ over the given dictionary $\boldsymbol{A}$ to find the few atoms in $\boldsymbol{A}$ that best represent the test pixel $\boldsymbol{x}$. The recovery process implicitly leads to a competition between the two subspaces. Therefore, the recovered sparse representation is naturally discriminative. Once the sparse vector $\hat{\gamma}$ is obtained, the class of $x$ can be determined by comparing the residuals $r_{b}(\boldsymbol{x})=\left\|\boldsymbol{x}-\boldsymbol{A}_{b} \hat{\boldsymbol{\alpha}}\right\|_{2}$ and $r_{t}(\boldsymbol{x})=\left\|\boldsymbol{x}-\boldsymbol{A}_{t} \hat{\boldsymbol{\beta}}\right\|_{2}$, where $\hat{\boldsymbol{\alpha}}$ and $\hat{\boldsymbol{\beta}}$ represent the recovered sparse coefficients corresponding to the background and target dictionaries, respectively. In our approach, the output of detector is calculated by

$$
D(\boldsymbol{x})=r_{b}(\boldsymbol{x})-r_{t}(\boldsymbol{x}) .
$$

If $D(\boldsymbol{x})>\delta$ with $\delta$ being a prescribed threshold, then $\boldsymbol{x}$ is determined as a target pixel; otherwise, $\boldsymbol{x}$ is labeled as background.

Fig. 3 shows an example of sparse reconstruction of a background test sample and a comparison to the pseudo-inverse reconstruction. This example illustrates the advantage of $\ell_{0}$-norm in classification problems over the conventional $\ell_{2}$-norm. The pseudo-inverse solution is obtained by solving the following minimum $\ell_{2}$-norm problem:

$$
\hat{\gamma}_{2}=\arg \min \|\boldsymbol{\gamma}\|_{2} \quad \text { subject to } \quad A \boldsymbol{\gamma}=\boldsymbol{x} .
$$

The above problem in (15), for the underdetermined linear system $\boldsymbol{A} \boldsymbol{\gamma}=\boldsymbol{x}$, has a closed-form solution $\hat{\boldsymbol{\gamma}}_{2}=\boldsymbol{A}^{\dagger} \boldsymbol{x}$ with $\boldsymbol{A}^{\dagger}$ being the pseudo-inverse of $\boldsymbol{A}$. For a test sample $\boldsymbol{x}$ and training dictionary $\boldsymbol{A}$, the minimum $\ell_{0}$-norm vector $\hat{\gamma}$ and minimum $\ell_{2}$-norm vector $\hat{\boldsymbol{\gamma}}_{2}$ are shown in Figs. 3(a) and (b), respectively. Blue and red represent entries corresponding to the background and target dictionaries, respectively. The original test sample $x$ and the partial reconstructed pixels using only the background dictionary $\hat{\boldsymbol{x}}_{b}=\boldsymbol{A}_{b} \hat{\boldsymbol{\alpha}}, \hat{\boldsymbol{x}}_{b, 2}=\boldsymbol{A}_{b} \hat{\boldsymbol{\alpha}}_{2}$ and only the target dictionary $\hat{\boldsymbol{x}}_{t}=\boldsymbol{A}_{t} \hat{\boldsymbol{\beta}}, \hat{\boldsymbol{x}}_{t, 2}=\boldsymbol{A}_{t} \hat{\boldsymbol{\beta}}_{2}$ are shown in Figs. 3(c) and (d). Although the pseudo-inverse solution $\hat{\gamma}_{2}$ yields perfect reconstruction, we see that it is not sparse and its nonzero entries spread over both classes. Thus, $\hat{\gamma}_{2}$ cannot be used directly for detection. The minimum $\ell_{0}$-norm solution $\hat{\gamma}$, on the contrary, has all of its nonzero entries concentrated in the background part, which indicates that the test sample lies in the background subspace. Furthermore, with the pseudo-inverse solution $\hat{\gamma}_{2}$, as seen in Fig. 3(d), neither $\boldsymbol{A}_{b} \hat{\boldsymbol{\alpha}}_{2}$ nor $\boldsymbol{A}_{t} \hat{\boldsymbol{\beta}}_{2}$ accurately approximates the original pixel, leading to a small difference between the residuals $r_{b, 2}(\boldsymbol{x})=0.568$ and $r_{t, 2}(\boldsymbol{x})=0.516$. Hence, the solution $\hat{\gamma}_{2}$ cannot be used to determine the class of the input solely based on the residuals. On the other hand, the residuals associated with the minimum $\ell_{0}$-norm solution $\hat{\gamma}$ are $r_{b}(\boldsymbol{x})=0.054 \ll r_{t}(\boldsymbol{x})=1$ (i.e., the original pixel $\boldsymbol{x}$ is well approximated by the background dictionary). Clearly, $\boldsymbol{x}$ is a background pixel using the minimum $\ell_{0}$-norm solution.
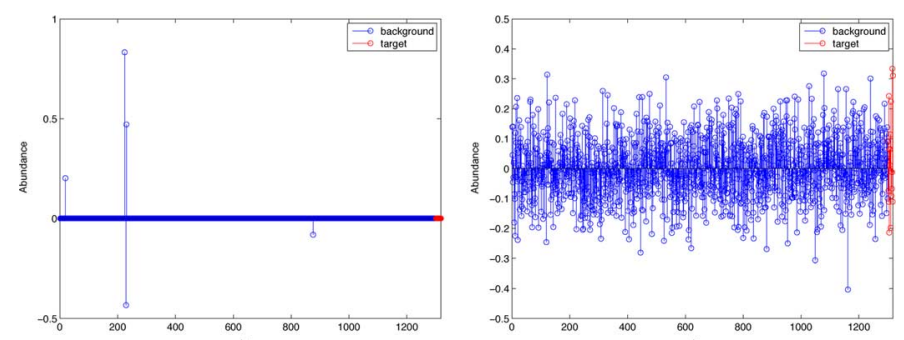

(a)

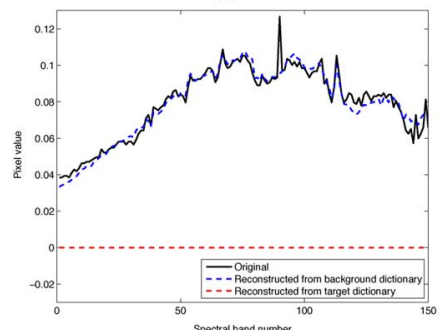

(c)

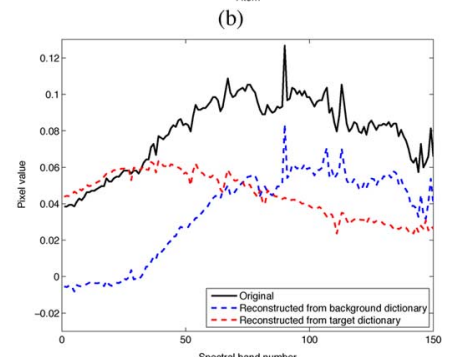

(d)

Fig. 3. Example of sparse reconstruction of a background test sample with a comparison to the minimum $\ell_{2}$-norm (pseudoinverse) solution. (a) Minimum $\ell_{0}$-norm solution $\hat{\gamma}$. (b) Pseudo-inverse solution $\hat{\gamma}_{2}=A^{\dagger} \boldsymbol{x}$. (c) Minimum $\ell_{0}$-norm reconstruction from the background dictionary $\hat{\boldsymbol{x}}_{b}=\boldsymbol{A}_{b} \hat{\boldsymbol{\alpha}}$ (blue dashed), reconstruction from the target dictionary $\hat{\boldsymbol{x}}_{t}=\boldsymbol{A}_{t} \hat{\boldsymbol{\beta}}$ (red dashed), and the original test sample $\boldsymbol{x}$ (black solid). (d) Pseudo-inverse reconstruction from the background dictionary $\hat{\boldsymbol{x}}_{b, 2}=\boldsymbol{A}_{b} \hat{\boldsymbol{\alpha}}_{2}$ (blue dashed), reconstruction from the target dictionary $\hat{\boldsymbol{x}}_{t, 2}=\boldsymbol{A}_{t} \hat{\boldsymbol{\beta}}_{2}$ (red dashed), and the original test sample $\boldsymbol{x}$ (black solid).

\section{Background and Target Dictionary Construction}

Another aspect of the problem that requires careful attention is how to construct appropriate dictionaries $\boldsymbol{A}_{b}$ and $\boldsymbol{A}_{t}$. Global dictionaries for target and background can be designed using given training data. However, in target detection applications there is usually a lack of training data especially for the target. The background is often modeled by a subspace by using some random pixels from the test image. Furthermore, a single target spectral signature, as employed in SMF, is often insufficient to represent a target material as the spectrum is affected by environmental conditions (e.g., illumination and atmospheric variations). By using physical models and the MORTRAN atmospheric-modeling program [34], meaningful target spectral signatures can be generated which can capture the target signature appearance over a wide range of atmospheric conditions. For example, in [21] a target subspace was constructed by generating a large number of target signatures using MORTRAN under various atmospheric conditions. A similar idea can be incorporated in our approach to construct a redundant target dictionary which could be invariant to the environmental variations. Furthermore, it can be combined with the idea of frame generation [35], [36] by imposing the constraints on tightness, maximum robustness, equiangularity, etc., to design more desirable overcomplete dictionaries. The K-SVD dictionary design technique [37], which alternately minimizes sparsity of the representation and updates the codebook to better fit the data, can also be used to form the redundant dictionaries to further improve the performance of the proposed sparsity-based algorithm.

In this paper, we use a small global target dictionary constructed by using some of the target pixels on one of the targets in the scene. For the background dictionary, instead of using 


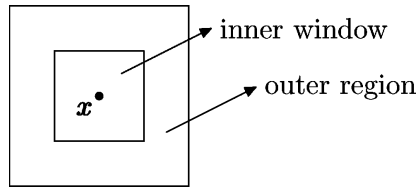

Fig. 4. Dual window centered at test sample $\boldsymbol{x}$.

a fixed global background dictionary containing samples from various background materials (e.g., trees, grass, road, buildings, etc.), we use an adaptive local background dictionary in order to better represent and capture the spectral signature of test sample. Specifically, the background dictionary $\boldsymbol{A}_{b}$ is generated locally for each test pixel using a dual window centered at the pixel of interest, as shown in Fig. 4. The inner window should be larger than the size of a target. Only pixels in the outer region form the atoms in $\boldsymbol{A}_{b}$. In this way, the subspace spanned by the background dictionary becomes adaptive to the local statistics. Therefore, if the test sample is a background pixel, it is highly likely that it finds very similar spectral characteristic in the background dictionary. On the other hand, if the test sample is a target pixel, it would be difficult for the pixel to find a good match in $\boldsymbol{A}_{b}$ since the outer window region does not include any target pixels. The usage of a dual window significantly improves the detection performance over a global background dictionary, as is shown via the simulation results in Section IV.

\section{Detection With Smoothing Constraint}

In the above process, the sparsity-based target detector is applied to each pixel in the test region independently without considering the correlation between neighboring pixels. Hyperspectral imagery, however, is usually smooth in the sense that neighboring pixels usually consist of similar materials and have similar spectral characteristics where small differences are often due to sensor noise and/or atmospheric variation. In this paper, we assume that there are multiple pixels on the target. Therefore, we propose to incorporate a smoothing penalty term in the proposed sparsity-based detector in order to exploit the spatial correlation between neighboring pixels.

Let $\boldsymbol{x}_{1}$ be a pixel of interest in a hyperspectral image $\boldsymbol{I}$, and $\boldsymbol{x}_{i}, i=2, \ldots, 5$ be its four nearest neighbors in the spatial domain, as shown in Fig. 5. While searching for the sparsest representation of the test sample $\boldsymbol{x}_{1}$, we simultaneously minimize the vector Laplacian at the reconstructed pixel $\hat{\boldsymbol{x}}_{1}$, which is a $B$-dimensional vector calculated as

$$
\begin{aligned}
\nabla^{2}\left(\hat{\boldsymbol{x}}_{1}\right) & =4 \hat{\boldsymbol{x}}_{1}-\hat{\boldsymbol{x}}_{2}-\hat{\boldsymbol{x}}_{3}-\hat{\boldsymbol{x}}_{4}-\hat{\boldsymbol{x}}_{5} \\
& =\boldsymbol{A}\left(4 \hat{\boldsymbol{\gamma}}_{1}-\hat{\boldsymbol{\gamma}}_{2}-\hat{\boldsymbol{\gamma}}_{3}-\hat{\boldsymbol{\gamma}}_{4}-\hat{\boldsymbol{\gamma}}_{5}\right)
\end{aligned}
$$

where $\hat{\boldsymbol{x}}_{i}=\boldsymbol{A} \hat{\boldsymbol{\gamma}}_{i}$ is the reconstruction of $\boldsymbol{x}_{i}$ and $\hat{\boldsymbol{\gamma}}_{i}$ is the corresponding recovered sparse vector. In this way, the reconstructed test sample is forced to have a similar spectral characteristics as its four nearest neighbors; hence, smoothness is enforced across the spectral pixels in the reconstructed image.

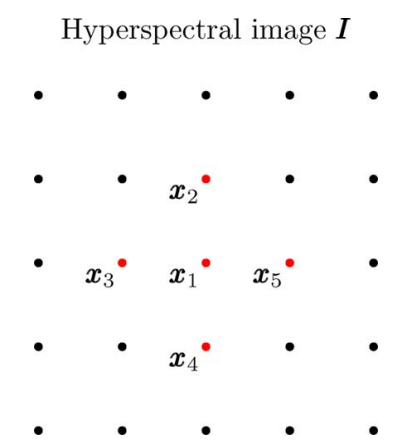

Fig. 5. Four nearest neighbors of a pixel $x_{1}$.

Let $\boldsymbol{\gamma}_{i}$ be the sparse vector associated with $\boldsymbol{x}_{i}$ (i.e., $\boldsymbol{x}_{i}=\boldsymbol{A} \boldsymbol{\gamma}_{i}$ ). The new problem with the smoothing constraint can now be formulated as

$$
\begin{aligned}
\operatorname{minimize} & \sum_{i=1}^{5}\left\|\boldsymbol{\gamma}_{i}\right\|_{0} \\
\text { subject to: } & A\left(4 \boldsymbol{\gamma}_{1}-\boldsymbol{\gamma}_{2}-\boldsymbol{\gamma}_{3}-\boldsymbol{\gamma}_{4}-\boldsymbol{\gamma}_{5}\right)=\mathbf{0} \\
& \boldsymbol{x}_{\boldsymbol{i}}=\boldsymbol{A} \boldsymbol{\gamma}_{i}, \quad i=1, \ldots, 5 .
\end{aligned}
$$

In (17), we aim to find the sparsest vector that approximately satisfies two sets of linear constraints. The first set forces the vector Laplacian of the reconstructed pixel $\hat{\boldsymbol{x}}_{1}$ to be minimal such that the reconstructed neighboring pixels have similar spectral characteristics, and the second set minimizes reconstruction errors. Now denote the concatenation of $\boldsymbol{\gamma}_{i}$ 's and $\boldsymbol{x}_{i}$ 's by

$$
\boldsymbol{\gamma}=\left[\begin{array}{c}
\gamma_{1} \\
\vdots \\
\gamma_{5}
\end{array}\right] \quad \text { and } \quad x=\left[\begin{array}{c}
x_{1} \\
\vdots \\
x_{5}
\end{array}\right]
$$

The linear constraints can be written in terms of $\boldsymbol{x}$ and $\boldsymbol{\gamma}$ as

$$
\begin{aligned}
& {\left[\begin{array}{lllll}
4 \boldsymbol{A} & -\boldsymbol{A} & -\boldsymbol{A} & -\boldsymbol{A} & -\boldsymbol{A}
\end{array}\right] \boldsymbol{\gamma}=\mathbf{0},} \\
& {\left[\begin{array}{lll}
A & & 0 \\
& \ddots & \\
0 & & A
\end{array}\right] \gamma=x .}
\end{aligned}
$$

Therefore, the optimization problem in (17) can be reformulated as

$$
\begin{aligned}
\operatorname{minimize} & \|\boldsymbol{\gamma}\|_{0} \\
\text { subject to: } & \tilde{\boldsymbol{A}} \boldsymbol{\gamma}=\tilde{\boldsymbol{x}},
\end{aligned}
$$

where

$$
\tilde{\boldsymbol{A}}=\left[\begin{array}{ccccc}
4 \boldsymbol{A} & -\boldsymbol{A} & -\boldsymbol{A} & -\boldsymbol{A} & -\boldsymbol{A} \\
\boldsymbol{A} & & & & \\
& \boldsymbol{A} & & & \\
& & \boldsymbol{A} & \boldsymbol{A} & \\
& & & & \boldsymbol{A}
\end{array}\right] \text { and } \tilde{\boldsymbol{x}}=\left[\begin{array}{l}
0 \\
\boldsymbol{x}
\end{array}\right] .
$$

The problem in (20) is the standard form of a linearly constrained sparsity-minimization problem and can be solved using the greedy SP algorithm [31]. Similar to the previous case in 


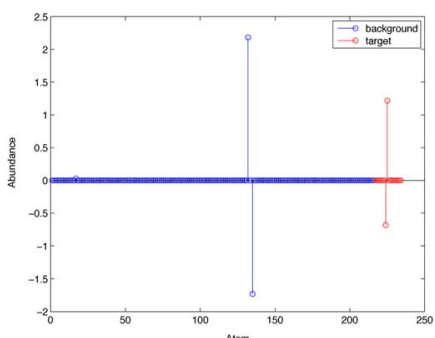

(a)

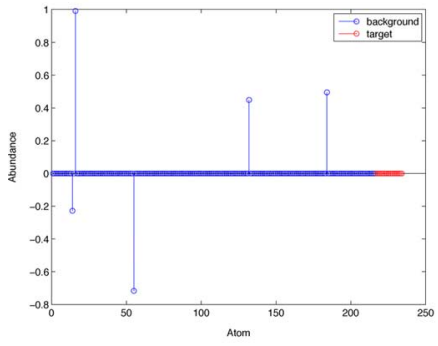

(c)

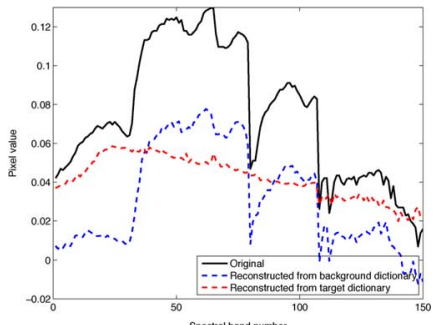

(b)

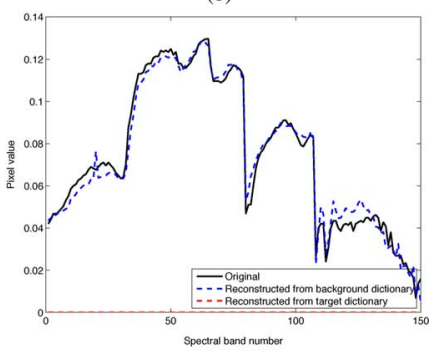

(d)
Fig. 6. Example comparing the reconstruction and detection problem for a background test sample without and with the smoothing constraint. (a) Solution to (11) (without smoothing constraint). (b) By solving (11), the reconstruction from the background dictionary (blue dashed), reconstruction from the target dictionary (red dashed), and the original test sample (black solid). (c) Solution to (20) (with smoothing constraint) for the centered test sample. (d) By solving (20), the reconstruction from the background dictionary (blue dashed), reconstruction from the target dictionary (red dashed), and the original test sample (black solid).

(11), this problem can also be relaxed to allow for approximation errors in empirical data and be rewritten as

$$
\hat{\boldsymbol{\gamma}}=\arg \min \|\boldsymbol{\gamma}\|_{0} \quad \text { subject to } \quad\|\tilde{\boldsymbol{A}} \boldsymbol{\gamma}-\tilde{\boldsymbol{x}}\|_{2} \leq \sigma
$$

or

$$
\hat{\boldsymbol{\gamma}}=\arg \min \|\tilde{\boldsymbol{A}} \boldsymbol{\gamma}-\tilde{\boldsymbol{x}}\|_{2} \quad \text { subject to } \quad\|\boldsymbol{\gamma}\|_{0} \leq K_{0}
$$

where $\sigma$ is the error tolerance and $K_{0}$ is the sparsity level.

By exploiting the smoothness across the HSI pixels, the detection performance can be significantly improved. Fig. 6 shows an example of a background test sample which is misclassified as a target using (11), but is correctly labeled using (20) with the smoothing constraint. The solution to (11) for the given test sample $\boldsymbol{x}$ is depicted in Fig. 6(a). We see that the nonzero entries of the solution correspond to both background and target training atoms, and the residuals are $r_{b}=0.5381, r_{t}=0.5088$. In the case with the smoothing constraint, by solving (20), the nonzero entries only concentrate on part corresponding to the background dictionary, and the residuals are $r_{b}=0.0538, r_{t}=1$. Clearly, $r_{b} \ll r_{t}$ and the test sample will thus be correctly labeled as a background sample.

Once the sparse vector in (20) is obtained, detection can be performed based on the characteristics of the sparse coefficients as it was done in Section III-B. We calculate the total residuals obtained separately from the target and background dictionaries

$$
r_{b}\left(\boldsymbol{x}_{1}\right)=\sqrt{\sum_{i=1}^{5}\left\|\boldsymbol{x}_{i}-\boldsymbol{A}_{b} \hat{\boldsymbol{\alpha}}_{i}\right\|_{2}^{2}}
$$

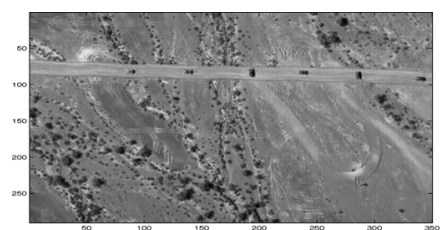

(a)

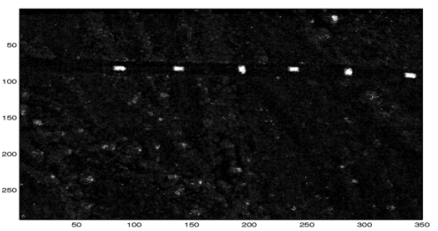

(c)

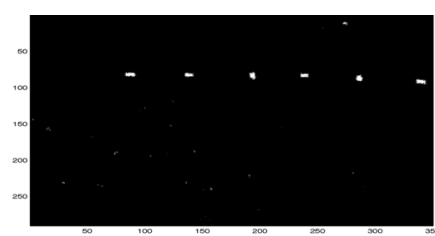

(b)

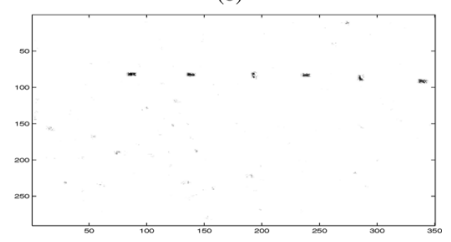

(d)
Fig. 7. Results for Desert Radiance II (DR-II) from (20) with the smoothing constraint. (a) Averaged image over 150 bands. (b) Sparsity-based target detector output: difference between $r_{b}$ and $r_{t}$. (c) Residual $r_{b}$ corresponding to the local background dictionary using the dual-window approach. (d) Residual $r_{t}$ corresponding to the target dictionary.

and

$$
r_{t}\left(\boldsymbol{x}_{1}\right)=\sqrt{\sum_{i=1}^{5}\left\|\boldsymbol{x}_{i}-\boldsymbol{A}_{t} \hat{\boldsymbol{\beta}}_{i}\right\|_{2}^{2}}
$$

where $\hat{\boldsymbol{\alpha}}_{i}$ and $\hat{\boldsymbol{\beta}}_{i}$ denote the recovered sparse coefficients for $\boldsymbol{x}_{i}$ associated with the background and target dictionaries, respectively. The output of the proposed sparsity-based detector for the center pixel $\boldsymbol{x}_{1}$ is computed by the difference of residuals and the detection decision is made in a similar fashion as in the other algorithms introduced in Section II:

$$
D\left(\boldsymbol{x}_{1}\right)=r_{b}\left(\boldsymbol{x}_{1}\right)-r_{t}\left(\boldsymbol{x}_{1}\right) \underset{H_{0}}{\stackrel{H_{1}}{\gtrless}} \delta .
$$

That is, if the output $D\left(\boldsymbol{x}_{1}\right)$ is greater than a prescribed threshold $\delta$, then the test sample $\boldsymbol{x}_{1}$ is labeled as a target; otherwise it is labeled as background.

\section{Simulation Results AND ANALYsis}

The proposed target detection algorithm, as well as the SMF, MSD, ASD, and SVM, are applied to several real HSI, and the results are compared both visually and quantitatively by the receiver operating characteristics (ROC) curves. The ROC curve describes the probability of detection (PD) as a function of the probability of false alarms (PFA). To be more specific, we pick thousands of different thresholds between the minimal and maximal values of the detector output. The class labels for all pixels in the test region are determined at each threshold. The PFA is calculated by the number of false alarms (background pixels determined as target) over the total number of pixels in the test region, and the $\mathrm{PD}$ is the ratio of the number of hits (target pixels determined as target) and the total number of true target pixels.

Two of the images, the desert radiance II data collection (DR-II) and forest radiance I data collection (FR-I), are from a hyperspectral digital imagery collection experiment (HYDICE) sensor [38]. The HYDICE sensor generates 210 bands across the whole spectral range from 0.4 to $2.5 \mu \mathrm{m}$ which includes the visible and short-wave infrared bands. We use 150 of the 210 bands (23rd-101st, 109th-136th, and 152nd-194th), removing 


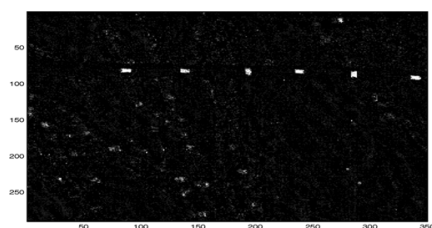

(a)

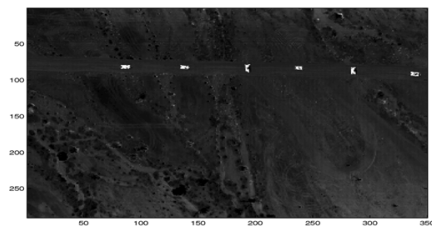

(c)

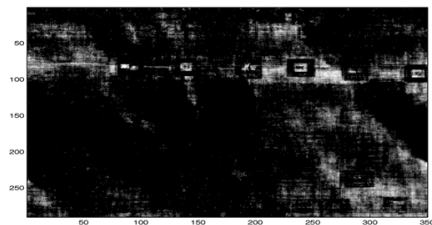

(e)

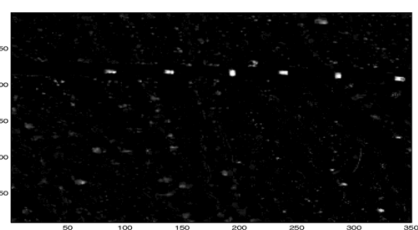

(b)

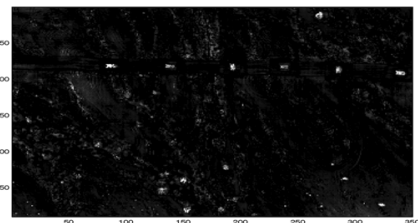

(d)

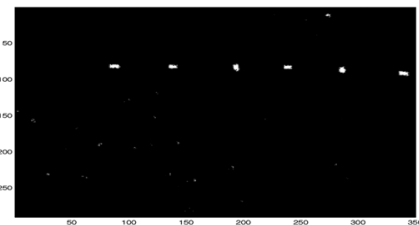

(f)
Fig. 8. Output for DR-II using local background dictionary (dual-window approach), with (a) sparsity-based target detector without smoothing constraint using (11), (b) SVM with composite kernel, (c) MSD, (d) SMF, and (e) ASD. (f) We repeat here the result of our proposed sparsity-based target detector with smoothing constraint for visual comparison.

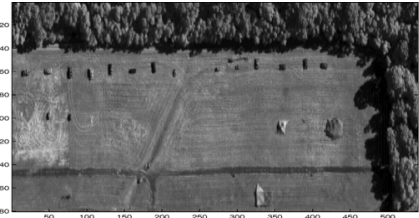

(a)

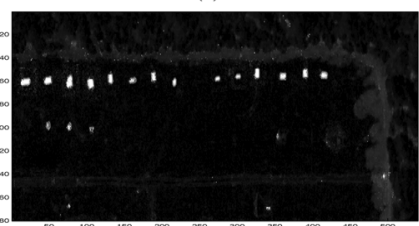

(c)

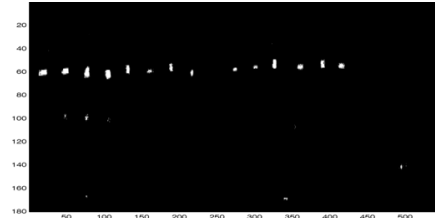

(b)

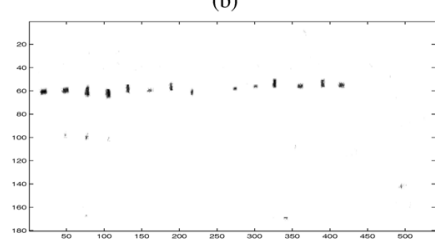

(d)
Fig. 9. Results for forest radiance I (FR-I) from (20) with smoothing constraint. (a) Averaged image over 150 bands. (b) Sparsity-based target detector output: difference between $r_{b}$ and $r_{t}$. (c) Residual $r_{b}$ corresponding to the background dictionary (dual-window approach). (d) Residual $r_{t}$ corresponding to the target dictionary.

the absorption and low-SNR bands. The DR-II image contains six military target on the dirt road and the FR-I image contains 14 targets along the tree line as depicted in Figs. 7(a) and 9(a), respectively. For these two HYDICE images, every pixel on the targets is considered a target pixel. The third image, collected from the Airborne Hyperspectral Imager (AHI) [39] operating in the long-wave infrared spectrum ranging from 8 to $11.5 \mu \mathrm{m}$, contains surface and buried mines as shown in Fig. 11(a), in which every pixel has 70 spectral bands. In this image, there are about 230 mines, each roughly of size $5 \times 5$ pixels and each mine is treated as a target when computing the PD.

For DR-II and FR-I, the spectral signatures of the target $\left\{\boldsymbol{a}_{i}^{t}\right\}_{i=1, \ldots, N_{t}}$ are collected directly from $N_{t}=18$ pixels from the leftmost target in the given hyperspectral data. The background signatures $\left\{\boldsymbol{a}_{i}^{b}\right\}_{i=1, \ldots, N_{b}}$ are generated by the pixels in the outer region of a dual window as discussed in

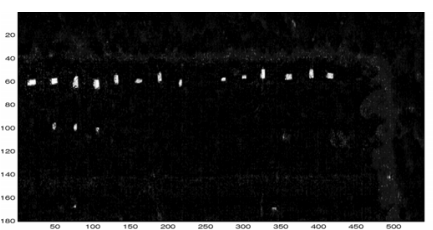

(a)

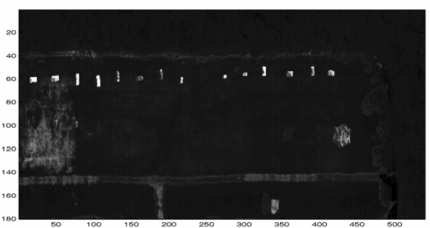

(c)

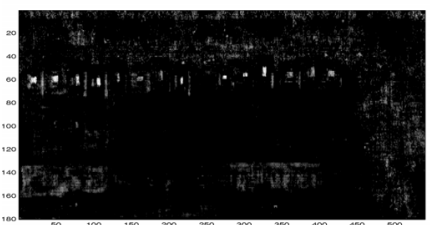

(e)

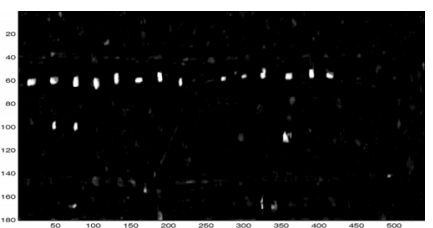

(b)

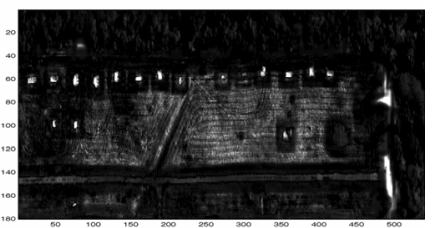

(d)

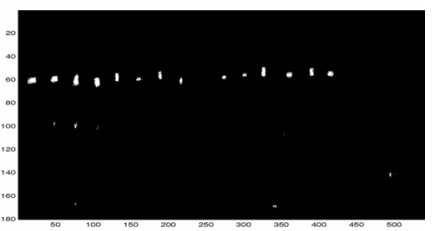

(f)
Fig. 10. Output for FR-I using local background dictionary (dual-window approach), with (a) sparsity-based target detector without smoothing constraint using (11), (b) SVM with composite kernel, (c) MSD, (d) SMF, and (e) ASD. (f) We repeat here the result of our proposed sparsity-based target detector with smoothing constraint for visual comparison.

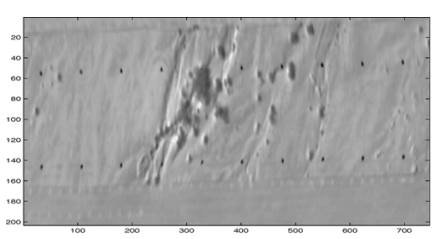

(a)

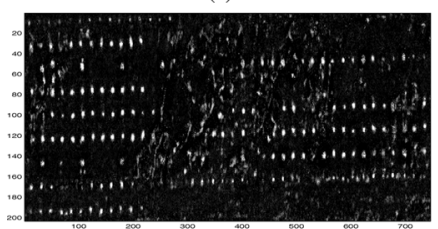

(c)

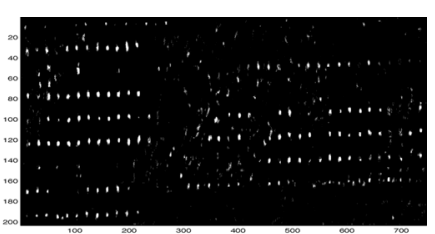

(b)

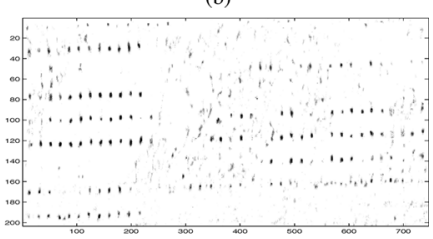

(d)
Fig. 11. Results for the mine image from (20) with smoothing constraint. (a) Averaged image over 70 bands. (b) Detector output: difference between $r_{b}$ and $r_{t}$. (c) Residual $r_{b}$ corresponding to the background dictionary (dual-window approach). (d) Residual $r_{t}$ corresponding to the target dictionary.

Section III. The size of the outer and inner windows are $21 \times 21$ and $15 \times 15$, respectively, and there are $N_{b}=216$ background training samples. The subspace pursuit algorithm [31] is used to solve the sparsity-constrained problems (11) and (20). The results of the proposed detector with the smoothing constraint for DR-II are shown in Fig. 7(b)-(d). Fig. 7(c) and (d) shows the residuals corresponding to the background dictionary $r_{b}(\boldsymbol{x})=\left\|\boldsymbol{x}-\boldsymbol{A}_{b} \hat{\boldsymbol{\alpha}}\right\|_{2}$, and the residual corresponding to the target dictionary $r_{t}(\boldsymbol{x})=\left\|\boldsymbol{x}-\boldsymbol{A}_{t} \hat{\boldsymbol{\beta}}\right\|_{2}$, respectively, whereas Fig. 7(b) shows the difference between $r_{b}$ and $r_{t}$. In Fig. 7(c), while background pixels are dark, the target pixels are bright due to the fact that for each target pixel the sparsity-constrained optimizer could not find good matches from the background dictionary; therefore, the sparse vector $\hat{\boldsymbol{\alpha}} \approx \mathbf{0}$ and the residual associated with the background dictionary is $r_{b}(\boldsymbol{x}) \approx\|\boldsymbol{x}\|$. On the contrary, in Fig. 7(d), the targets are dark while the background are bright. Finally, as shown in Fig. 7(b), the difference between $r_{b}$ and $r_{t}$ will further suppress the background and 


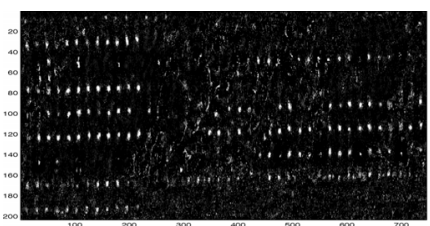

(a)

(c)

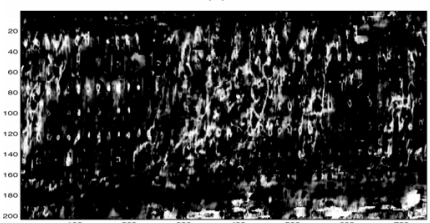

(e)

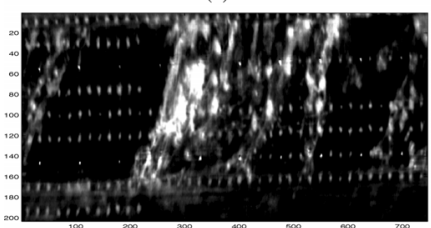

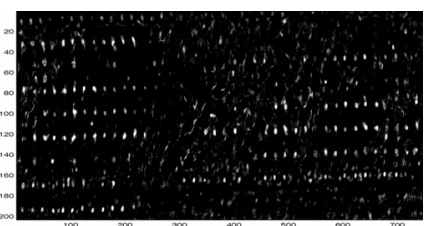

(b)

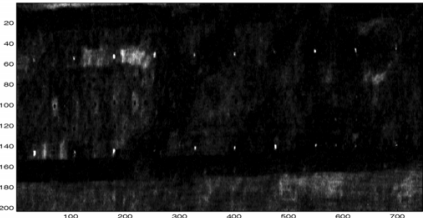

(d)

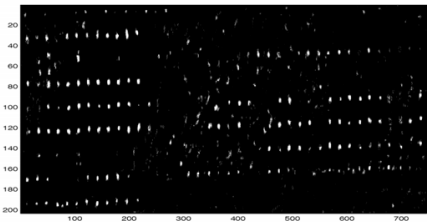

(f)
Fig. 12. Output for the mine image using local background dictionary (dualwindow approach), with (a) sparsity-based target detector without smoothing constraint, (b) SVM with composite kernel, (c) MSD, (d) SMF, and (e) ASD. (f) We repeat here the result of our proposed sparsity-based target detector with smoothing constraint for visual comparison.

emphasizes the targets, thus yielding better detection performance. Similar results can be seen in Fig. 9(b)-(d) for the FR-I image. In Fig. 9(c) which represents the residual image $r_{b}$, although the targets are bright, we can also see the shadow of trees near the upper and right borders of the image has higher magnitude than the other background areas. In Fig. 9(b), the shadow is suppressed and this improves the false alarm rate.

Similar results can be observed in Fig. 11 for the mine image, where the target dictionary $\boldsymbol{A}_{t}$ is generated from $N_{t}=50$ training samples of two mines, each occupying a $5 \times 5$ area, outside the test region. Since the targets in this image are smaller than that of the two HYDICE images, the inner window size is chosen to be $9 \times 9$ and the outer window size remains $21 \times 21$. The background dictionary $\boldsymbol{A}_{b}$ then consists of $N_{b}=360$ samples.

Next we demonstrate the importance of employing a locally adaptive background dictionary. The sparsity-based target detection algorithm is applied to the DR-II and FR-I images using local and global background dictionaries. The local $\boldsymbol{A}_{b}$ is generated by $N_{b}=216$ pixels in the outer region of the dual window centered at the test sample as in Fig. 4, and the global dictionary ( $N_{b}=1300$ for DR-II and $N_{b}=1656$ for FR-I) is generated by randomly collecting background pixels, which can be reduced to a smaller size by an unsupervised clustering algorithm such as $\mathrm{K}$-means. The detection performance is significantly improved by using a local dictionary, as seen in the ROC curves shown in Fig. 13. This is because a fixed global dictionary fails to capture the local similarity between pixels in a small neighborhood. A local dictionary exploits the local statistics and helps to find better resemblance of test samples. We see in Fig. 13 that the detector using local dictionaries outperforms the one using global dictionaries by a large margin for both HYDICE images.

Under the same settings (i.e., same target and background training samples for all detectors), we compare the performance

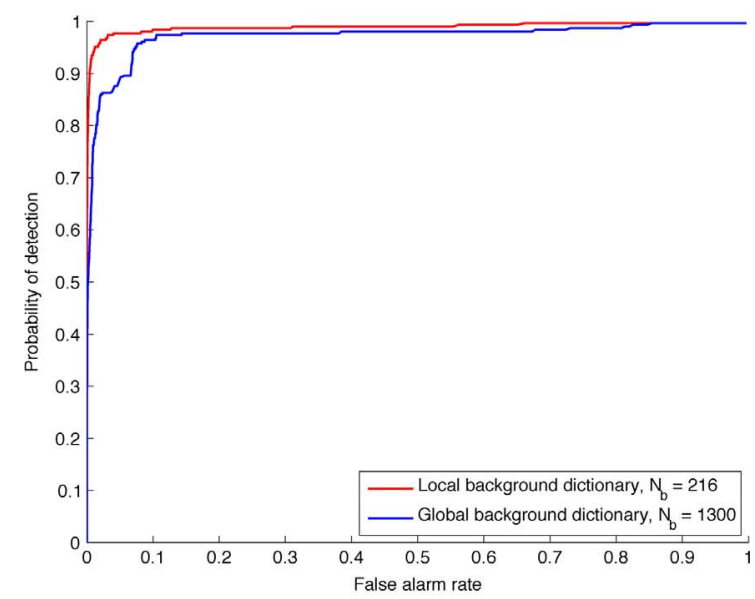

(a)

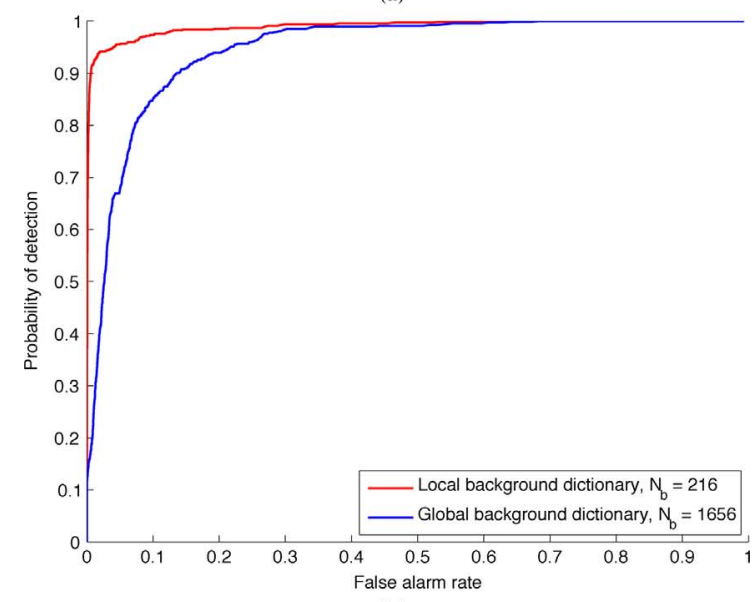

(b)

Fig. 13. ROC curves using the sparsity-based target detector with smoothing constraint for (a) DR-II and (b) FR-I with local and global background dictionaries.

of the proposed sparsity-based algorithm to the previously developed conventional classifier SVM and detectors MSD, SMF, ASD using both global and local background dictionaries. Let $\boldsymbol{A}_{t}=\left[\begin{array}{lll}\boldsymbol{a}_{1}^{t} & \cdots & \boldsymbol{a}_{N_{t}}^{t}\end{array}\right]$ and $\boldsymbol{A}_{b}=\left[\begin{array}{lll}\boldsymbol{a}_{1}^{b} & \cdots & \boldsymbol{a}_{N_{b}}^{b}\end{array}\right]$ be, respectively, the target and background dictionaries used in the proposed sparsity-based algorithm. Note that in the local case, $A_{b}$ is adaptive and changes for each test pixel. In order to have a fair comparison, in the case of SMF the target signature is the mean of the target dictionary atoms $\left\{\boldsymbol{a}_{i}^{t}\right\}_{i=1, \ldots, N_{t}}$ and the background covariance is obtained from the background dictionary $A_{b}$. In the SMF implementation, a regularization term is added to the background covariance matrix such that the inverse matrix $\hat{\boldsymbol{C}}^{-1}$ in (3) is more stable, as described in [27]. In the case of MSD, the eigenvectors corresponding to the significant eigenvalues of the covariance matrices obtained from atoms in $\boldsymbol{A}_{t}$ and $A_{b}$ are used to generate the basis for the target and background subspaces, respectively [23]. For ASD, the basis for target subspace are generated in the same way as in MSD. The ASD noise covariance matrix is computed from the background training samples $\left\{\boldsymbol{a}_{i}^{b}\right\}_{i=1, \ldots, N_{b}}$ and a regularization term is added to the noise covariance matrix in order to obtain a stable inverse matrix. In SVM, a model is trained using atoms in $\boldsymbol{A}_{b}$ and $\boldsymbol{A}_{t}$ as two different classes using a composite kernel which combines the spectral and spatial feature via a weighted summation, where $K_{s}$ 


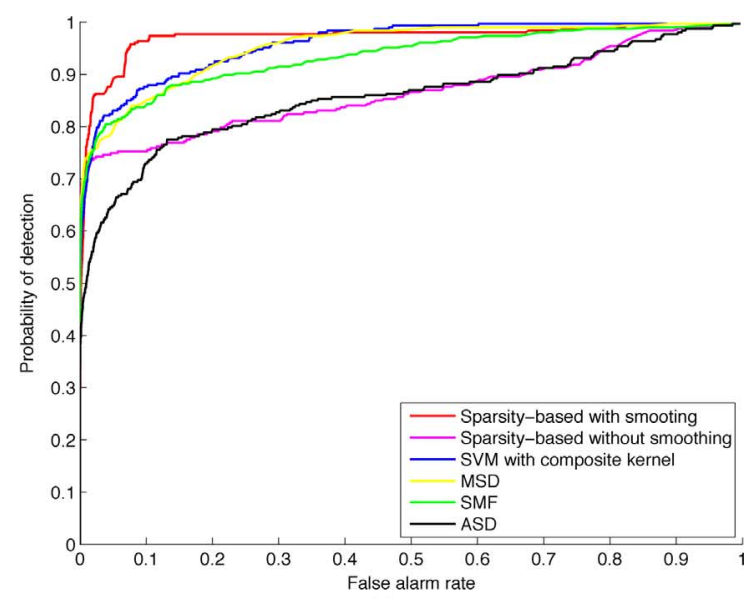

(a)

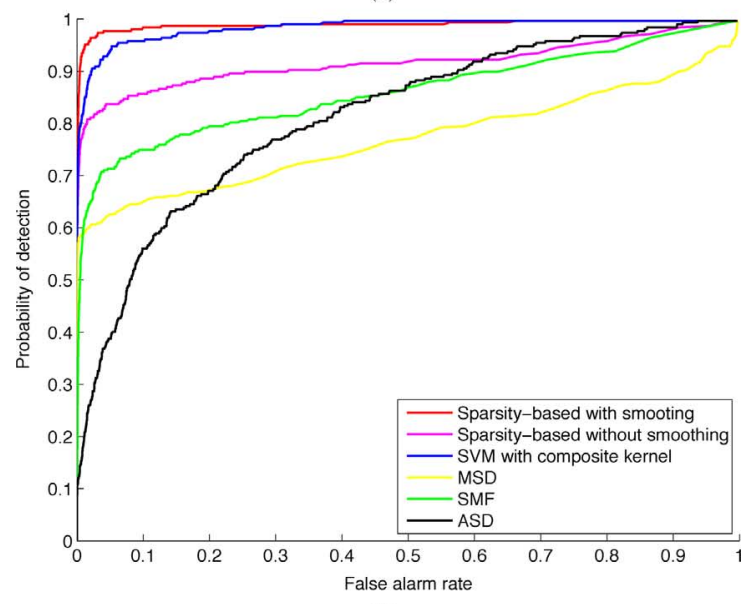

(b)

Fig. 14. ROC curves for DR-II. (a) Global background dictionary, $N_{b}=1300$. (b) local background dictionary (dual-window approach), $N_{b}=216$.

and $K_{w}$ in (1) are radial basis function kernels [9]. All parameters are adjusted to obtain the best possible performance. Under the current setting of target and background dictionaries, the proposed detector has computational complexity comparable to that of the classical detectors SMF, MSD, and ASD.

The ROC curves in both the global and local cases for DR-II are shown in Fig. 14. We see that the sparsity-based detector with the smoothing term using a local background dictionary outperforms all other detectors. The SMF performs poorly since the target signature is represented by a single vector, while in all other approaches the targets are assumed to approximately lie in a subspace. For visual comparison, the detector outputs for SVM, MSD, SMF, and ASD are also displayed in Fig. 8, where the locally adaptive background dictionary is employed. One can immediately notice that the sparsity-based detector with the smoothing constraint also leads to the best visual quality.

The ROC curves for FR-I are shown in Fig. 15. The FR-I image is more difficult than the DR-II due to the presence of the trees and shadow whose spectral curves have some resemblance to that of the targets. From the ROC plots, the proposed algorithm still leads to the best performance. For visual inspection, the detection results obtained by SVM, MSD, SMF, and ASD are illustrated in Fig. 10. For all detectors in Fig. 10, we can see the bright spots in the shadow area along the tree line.

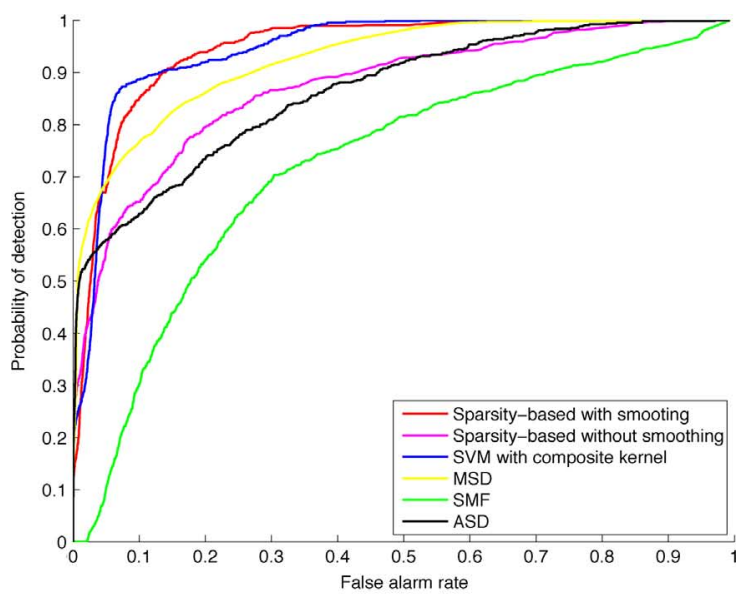

(a)

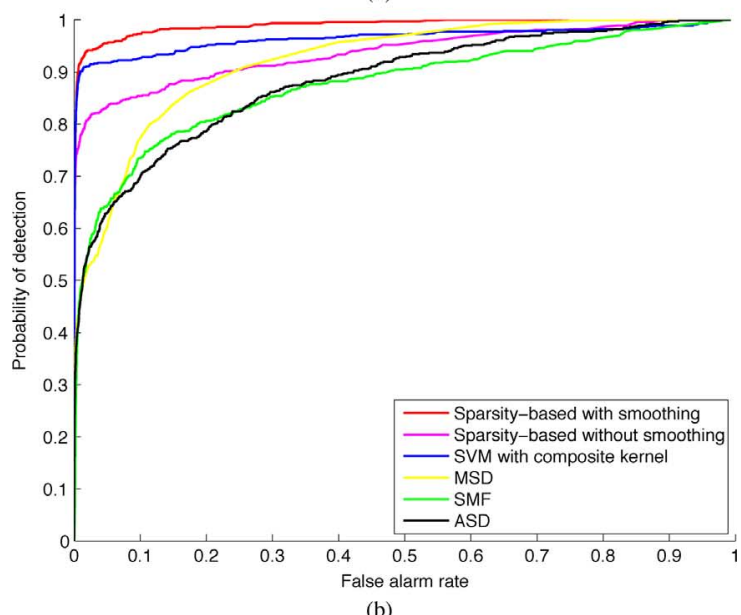

(b)

Fig. 15. ROC curves for FR-I. (a) Global background dictionary, $N_{b}=1656$. (b) local background dictionary (dual-window approach), $N_{b}=216$.

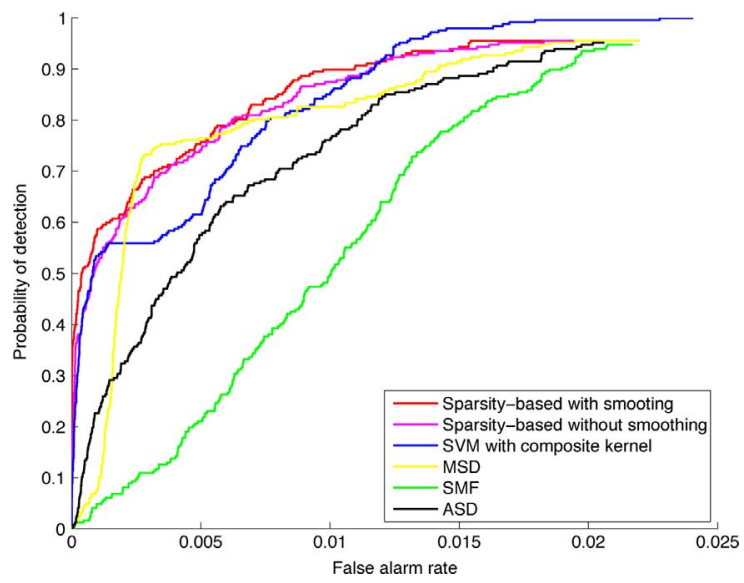

Fig. 16. ROC curves for the mine image using local background dictionary (dual-window approach), $N_{b}=360$.

This is alleviated by the proposed detection algorithm, as seen in Fig. 9(b).

The AHI image of mines is the most difficult one among the three test images. The targets include surface mines and buried mines that are invisible. In this case, the ROC curve is obtained slightly differently in that only one pixel on the mine needs to be correctly labeled for the mine to be declared as a target. Therefore, the PD is calculated by the number of hits divided 
by the total number of mines in the test region. In this experiment, for all detectors, we use $N_{t}=50$ target training samples from two mines outside the test region and $N_{b}=360$ background training samples adaptively constructed for each test pixel by the dual-window approach with inner and outer windows of size $9 \times 9$ and $21 \times 21$, respectively. The ROC curves for the mine image using local dictionaries are shown in Fig. 16. The proposed sparsity-based target detection algorithm still outperforms the other algorithms, especially at low PFA. The outputs for SVM, MSD, SMF, and ASD are displayed as images in Fig. 12. We see that although the MSD yields higher PD at certain PFA, there is a large background area in the middle of the image where pixels have very high magnitude, hence increasing the number of false alarms.

\section{CONCLUSION}

In this paper, we propose a target detection algorithm for hyperspectral imagery based on sparse representation of the test samples. In the proposed algorithm, the sparse representation is recovered by solving a constrained optimization problem that simultaneously addresses the sparsity constraint, reconstruction accuracy, and a smoothness penalty on the reconstructed image. Detection decision is obtained from the recovered sparse vectors by reconstruction. The new algorithm consistently outperforms the previously developed detectors in terms of both qualitative and quantitative measures, as demonstrated by experimental results in several real hyperspectral images. Future research includes the construction of better dictionaries. For example, the proposed detector can be improved by generating dictionaries invariant to the effect of atmospheric absorption [21]. We will also investigate the design and exploitation of more discriminative dictionaries learned from the training data [37], [40].

\section{REFERENCES}

[1] M. Borengasser, W. S. Hungate, and R. Watkins, Hyperspectral Remote Sensing_Principles and Applications. Boca Raton, FL: CRC, 2008.

[2] D. Manolakis and G. Shaw, "Detection algorithms for hyperspectral imaging applications," IEEE Signal Process. Mag., vol. 19, no. 1, pp. 29-43, Jan. 2002.

[3] D. W. J. Stein, S. G. Beaven, L. E. Hoff, E. M. Winter, A. P. Schaum, and A. D. Stocker, "Anomaly detection from hyperspectral imagery," IEEE Signal Process. Mag., vol. 19, no. 1, pp. 58-69, Jan. 2002.

[4] M. T. Eismann, A. D. Stocker, and N. M. Nasrabadi, "Automated hyperspectral cueing for civilian search and rescue," Proc. IEEE, vol. 97, no. 6, pp. 1031-1055, Jun. 2009.

[5] N. K. Patel, C. Patnaik, S. Dutta, A. M. Shekh, and A. J. Dave, "Study of crop growth parameters using airborne imaging spectrometer data," Int. J. Remote Sens., vol. 22, no. 12, pp. 2401-2411, Aug. 2001.

[6] B. Datt, T. R. McVicar, T. G. Van Niel, D. L. B. Jupp, and J. S. Pearlman, "Preprocessing EO-1 hyperion hyperspectral data to support the application of agricultural indexes," IEEE Trans. Geosci. Remote Sens., vol. 41, no. 6, pp. 1246-1259, Jun. 2003.

[7] B. Hörig, F. Kühn, F. Oschütz, and F. Lehmann, "HyMap hyperspectral remote sensing to detect hydrocarbons," Int. J. Remote Sens., vol. 22, no. 8, pp. 1413-1422, 2001.

[8] B. E. Boser, I. M. Guyon, and V. N. Vapnik, "A training algorithm for optimal margin classifiers," in Proc. 5th Annu. Workshop Comput. Learn. Theory, 1992, pp. 144-152.

[9] V. N. Vapnik, The Nature of Statistical Learning Theory. New York: Springer, 1995.

[10] J. A. Gualtieri and R. F. Cromp, "Support vector machines for hyperspectral remote sensing classification," in Proc. SPIE, Jan. 1998, vol. 3584, pp. 221-232.
[11] F. Melgani and L. Bruzzone, "Classification of hyperspectral remote sensing images with support vector machines," IEEE Trans. Geosci. Remote Sens., vol. 42, no. 8, pp. 1778-1790, Aug. 2004.

[12] F. C. Robey, D. R. Fuhrmann, E. J. Kelly, and R. Nitzberg, "A CFAR adaptive matched filter detector," IEEE Trans. Aerosp. Electron. Syst., vol. 28, no. 1, pp. 208-216, Jan. 1992.

[13] D. Manolakis, G. Shaw, and N. Keshava, "Comparative analysis of hyperspectral adaptive matched filter detectors," in Proc. SPIE Conf. Algorithms for Multispectral, Hyperspectral, Ultraspectral Imagery VI, Apr. 2000, vol. 4049, pp. 2-17.

[14] L. L. Scharf and B. Friedlander, "Matched subspace detectors," IEEE Trans. Signal Process., vol. 42, no. 8, pp. 2146-2157, Aug. 1994.

[15] S. Kraut, L. L. Scharf, and L. T. McWhorter, "Adaptive subspace detectors," IEEE Trans. Signal Process., vol. 49, no. 1, pp. 1-16, Jan. 2001.

[16] J. Wright, A. Y. Yang, A. Ganesh, S. Sastry, and Y. Ma, "Robust face recognition via sparse representation," IEEE Trans. Pattern Anal. Mach. Intell., vol. 31, no. 2, pp. 210-227, Feb. 2009.

[17] R. Basri and D. W. Jacobs, "Lambertian reflectance and linear subspaces," IEEE Trans. Pattern Anal. Mach. Intell., vol. 25, no. 2, pp. 218-233, Feb. 2003.

[18] J. K. Pillai, V. M. Patel, and R. Chellappa, "Sparsity inspired selection and recognition of iris images," in Proc. IEEE 3rd Int. Conf. Biometrics: Theory, Applicat., Syst., Sep. 2009, pp. 1-6.

[19] X. Hang and F.-X. Wu, "Sparse representation for classification of tumors using gene expression data," J. Biomed. Biotechnol., vol. 2009, 2009, doi:10.1155/2009/403689.

[20] Z. Guo, T. Wittman, and S. Osher, "L1 unmixing and its application to hyperspectral image enhancement," in Proc. SPIE Conf. Algorithms Technol. Multispectral, Hyperspectral, Ultraspectral Imagery XV, Apr. 2009, vol. 7334.

[21] G. Healey and D. Slater, "Models and methods for automated material identification in hyperspectral imagery acquired under unknown illumination and atmospheric conditions," IEEE Trans. Geosci. Remote Sens., vol. 37, no. 6, pp. 2706-2717, Jun. 1999.

[22] B. Thai and G. Healey, "Invariant subpixel material detection in hyperspectral imagery," IEEE Trans. Geosci. Remote Sens., vol. 40, no. 3, pp. 599-608, Mar. 2002.

[23] H. Kwon and N. M. Nasrabadi, "A comparative analysis of kernel subspace target detectors for hyperspectral imagery," EURASIP J. Appl. Signal Process., vol. 2007, no. 1, pp. 193-193, Jan. 2007.

[24] G. Camps-Valls, L. Gomez-Chova, J. Muñoz-Marí, J. Vila-Francés, and J. Calpe-Maravilla, "Composite kernels for hyperspectral image classification," IEEE Geosci. Remote Sens. Lett., vol. 3, no. 1, pp. 93-97, Jan. 2006.

[25] A. Plaza, J. A. Benediktsson, J. W. Boardman, J. Brazile, L. Bruzzone, G. Camps-Valls, J. Chanussot, M. Fauvel, P. Gamba, A. Gualtieri, M. Marconcini, J. C. Tilton, and G. Trianni, "Recent advances in techniques for hyperspectral image processing," Remote Sens. Environ., vol. 113, pp. S110-S122, Sep. 2009, Supplement 1.

[26] B. Schlkopf and A. J. Smola, Learning With Kernels: Support Vector Machines, Regularization, Optimization, and Beyond (Adaptive Computation and Machine Learning). Cambridge, MA: MIT Press, 2001.

[27] N. M. Nasrabadi, "Regularized spectral matched filter for target recognition in hyperspectral imagery," IEEE Signal Process. Lett., vol. 15, pp. 317-320, 2008.

[28] D. L. Donoho, "For most large underdetermined systems of linear equations the minimal $l_{1}$-norm solution is also the sparsest solution," Comm. Pure Appl. Math., vol. 59, no. 6, pp. 797-829, 2006.

[29] A. M. Bruckstein, D. L. Donoho, and M. Elad, "From sparse solutions of systems of equations to sparse modeling of signals and images," SIAM Rev., vol. 51, no. 1, pp. 34-81, 2009.

[30] J. Tropp and A. Gilbert, "Signal recovery from random measurements via orthogonal matching pursuit," IEEE Trans. Inf. Theory, vol. 53, no. 12, pp. 4655-4666, Dec. 2007.

[31] W. Dai and O. Milenkovic, "Subspace pursuit for compressive sensing signal reconstruction," IEEE Trans. Inf. Theory, vol. 55, no. 5, pp. 2230-2249, May 2009.

[32] J. A. Tropp and S. J. Wright, "Computational methods for sparse solution of linear inverse problems," Proc. IEEE, vol. 98, no. 6, pp. 948-958, Jun. 2010.

[33] E. van den Berg and M. P. Friedlander, "Probing the Pareto frontier for basis pursuit solutions," SIAM J. Sci. Comput., vol. 31, no. 2, pp. 890-912, Nov. 2008. 
[34] A. Berk, L. Bernstein, and D. Robertson, Geophysics Laboratory, Bedford, MA, "MORTRAN: A moderate resolution model for LOWTRAN 7,” Tech. Rep. GL-TR-90-0122, 1989.

[35] J. Kovacevic and A. Chebira, "Life beyond bases: The advent of frames (Part I)," IEEE Signal Process. Mag., vol. 24, no. 4, pp. 86-104, Jul. 2007.

[36] J. Kovacevic and A. Chebira, "Life beyond bases: The advent of frames (Part II)," IEEE Signal Process. Mag., vol. 24, no. 5, pp. 115-125, Sep. 2007.

[37] M. Aharon, M. Elad, and A. M. Bruckstein, "K-SVD: An algorithm for designing overcomplete dictionaries for sparse representation," IEEE Trans. Signal Process., vol. 54, no. 11, pp. 4311-4322, Nov. 2006.

[38] R. W. Basedow, D. C. Carmer, and M. E. Anderson, "HYDICE system: Implementation and performance," in Proc. SPIE Conf. Algorithms Technol. Multispectral, Hyperspectral, and Ultraspectral Imagery XV, Apr. 1995, vol. 2480, pp. 258-267.

[39] P. G. Lucey, T. J. Williams, M. Mignard, J. Julian, D. Kobubun, G. Allen, D. Hampton, W. Schaff, M. J. Schlangenand, E. M. Winter, W. B. Kendall, A. D. Stocker, K. A. Horton, and A. P. Bowman, "AHI: An airborne long-wave infrared hyperspectral imager," in Proc. SPIE Conf. Airborne Reconnaissance XXII, 1998, vol. 3431, pp. 36-43.

[40] J. Mairal, M. Elad, and G. Sapiro, "Sparse representation for color image restoration," IEEE Trans. Pattern Anal. Mach. Intell., vol. 17, no. 1, pp. 53-69, Jan. 2008.

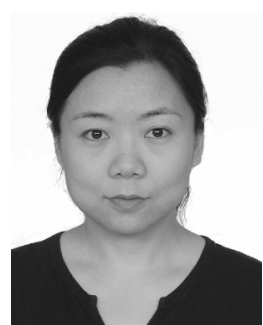

and filter banks.

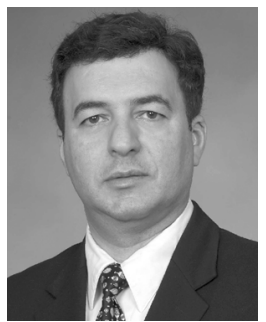

Nasser M. Nasrabadi (S'80-M'84-SM'92-F'01) received the B.Sc. (Eng.) and Ph.D. degrees in electrical engineering from Imperial College of Science and Technology, University of London, London, U.K., in 1980 and 1984, respectively.

From October 1984 to December 1984, he was with IBM (U.K.) as a Senior Programmer. From 1985 to 1986, he was with Philips Research Laboratory, Briarcliff Manor, NY, as a Member of Technical Staff. From 1986 to 1991, he was an Assistant Professor in the Department of Electrical Engineering, Worcester Polytechnic Institute, Worcester, MA. From 1991 to 1996, he was an Associate Professor with the Department of Electrical and Computer Engineering, State University of New York at Buffalo, Buffalo, NY. Since September 1996, he has been a Senior Research Scientist (ST) with the U.S. Army Research Laboratory (ARL), Adelphi, MD, working on image processing and automatic target recognition.

Dr. Nasrabadi has served as an Associate Editor for the IEEE TRANSACTIONS on IMAGE PROCESSING, the IEEE TRANSACTIONS ON CirCUITS, SySTEMS AND Video TeChNOLOGY, and the IEEE TRANSACTIONS ON NEURAL NeTWORKS. $\mathrm{He}$ is also a Fellow of ARL and SPIE. His current research interests are in Hyperspectral imaging, automatic target recognition, statistical machine learning theory, robotics, and neural networks applications to image processing.

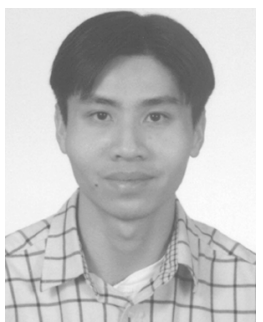

Trac D. Tran (S'94-M'98-SM'08) received the B.S. and M.S. degrees from the Massachusetts Institute of Technology, Cambridge, in 1993 and 1994, respectively, and the Ph.D. degree from the University of Wisconsin, Madison, in 1998, all in electrical engineering.

In July of 1998, he joined the Department of Electrical and Computer Engineering, The Johns Hopkins University, Baltimore, MD, where he currently holds the rank of Associate Professor. His research interests are in the field of digital signal processing, particularly in sparse representation, sparse recovery, sampling, multi-rate systems, filter banks, transforms, wavelets, and their applications in signal analysis, compression, processing, and communications. His pioneering research on integercoefficient transforms and pre-/post-filtering operators has been adopted as critical components of Microsoft Windows Media Video 9 and JPEG XR C the latest international still-image compression standard ISO/IEC 29199-2. In the summer of 2002, he was an ASEE/ONR Summer Faculty Research Fellow at the Naval Air Warfare Center C Weapons Division (NAWCWD) at China Lake, CA. He is currently a regular consultant for the U.S. Army Research Laboratory in Adelphi, MD.

Dr. Tran was the co-director (with Prof. J. L. Prince) of the 33rd Annual Conference on Information Sciences and Systems (CISS'99), Baltimore, MD, in March 1999. He has served as an Associate Editor of the IEEE TRAnsactions on Signal Processing as well as the IEEE TRANSACtions ON IMAge Processing. He was a former member of the IEEE Technical Committee on Signal Processing Theory and Methods (SPTM TC) and is a current member of the IEEE Image Video and Multidimensional Signal Processing (IVMSP) Technical Committee. He received the NSF CAREER award in 2001, the William H. Huggins Excellence in Teaching Award from The Johns Hopkins University in 2007, and the Capers and Marion McDonald Award for Excellence in Mentoring and Advising in 2009. 